\title{
NUEVOS REGISTROS DE CRAUGASTOR YUCATANENSIS (ANURA: CRAUGASTORIDAE) EN EL ESTADO DE YUCATÁN
}

NEW RECORDS OF CRAUGASTOR YUCATANENSIS (ANURA: CRAUGASTORIDAE) IN THE STATE OF YUCATAN Pedro E. Nahuat-Cervera ${ }^{1,2 *}$, Allam H. Tzab-PeCH ${ }^{3}$, Aarón Rodríguez-Blanco ${ }^{4}$ Juan Cruzado ${ }^{5}$

${ }^{1}$ Ekuneil Península de Yucatán. Calle 52, 670. Colonia Centro, C.P. 97000. Mérida, Yucatán, México.

${ }^{2}$ Asociación Juvenil de Ciencia Yucatán A.C. Calle 35-C por 60, Fraccionamiento Colonia Buenavista, C.P. 97215. Mérida, Yucatán, México.

${ }^{3}$ Calle 22, 121, Colonia Homún, C.P. 97580 . Homún, Yucatán, México.

${ }^{4}$ Campus de Ciencias Biológicas y Agropecuarias, Universidad Autónoma de Yucatán, Km 15.5. carr. Mérida-Xmatkuil, C.P.97315. Mérida, Yucatán, México.

5Calle 33, \#351, Fraccionamiento Francisco de Montejo, Mérida, Yucatán, C.P. 97203, México.

*Correspondencia: pedro.nahuat4@gmail.com

México: Yucatán: Municipio de Izamal, $6.4 \mathrm{~km}$ al este de Sitilpech $\left(20.930461^{\circ} \mathrm{N}, 88.894790^{\circ} \mathrm{O}\right.$, WGS $\left.84,36 \mathrm{msnm}\right)$, 31 de enero de 2020. Se observó un ejemplar adulto a las 19:30 h, justo después de una lluvia fuerte, la rana estaba activa sobre un cúmulo de rocas (Fig. IA) rodeadas por vegetación secundaria y remanentes de selva baja caducifolia, a aproximadamente $18 \mathrm{~m}$ de la entrada a una caverna. El ejemplar fue encontrado y fotografiado por Aarón Rodríguez-Blanco. La fotografía se depositó en la colección digital de The University of Texas at Arlington (UTADC 9547). Esta observación representa el registro más al norte de Craugastor yucatanensis, así como el primer registro en el municipio de Izamal, Yucatán, encontrándose a $43.8 \mathrm{~km}$ al noroeste del registro más cercano en la zona arqueológica de Chichén Itzá, municipio de Tinúm, Yucatán, México (CarbajalMárquez et al., 2019; Fig. 2).
México: Yucatán: Municipio de Homún, $2.6 \mathrm{~km}$ al este de Homún $\left(20.734568^{\circ} \mathrm{N}, 89.259454^{\circ} \mathrm{O}\right.$, WGS $\left.84,28 \mathrm{msnm}\right)$, 14 de marzo de $2020,18: 45$ h; $3.8 \mathrm{~km}$ al noreste de Homún $\left(20.741785^{\circ} \mathrm{N}, 89.247599^{\circ} \mathrm{O}\right.$, WGS 84$), 14$ de marzo de 2020 , 21:00 h. Ejemplares encontrados y fotografiados por Pedro E. Nahuat-Cervera, Allam H. Tzab-Pech y Juan Cruzado. Los cuatro ejemplares se encontraron al interior de cavernas. Al encontrar cada ejemplar, estos se fotografiaron in situ (Fig. IB-E), para posteriormente tomar datos morfológicos (Tabla 1), y las variables microambientales y del microhábitat (Tabla 2). Las fotografías se depositaron en la colección digital de The University of Texas at Arlington (UTADC 9548-9551). Estas observaciones representan los primeros registros de esta especie para el municipio de Homún, Yucatán, llenando un vacío de distribución entre las localidades conocidas más cercanas, $71 \mathrm{~km}$ al SO (Fig. 2), en las cavernas de Xpukil, municipio de

Table 1. Morphological data of the individuals of Craugastor yucatanensis reported in this note. Symbology: Snout to vent length (LHC), head width (AC), femur length (LF), foot length (LP), eardrum diameter (DT), eye diameter (DO).

Tabla 1. Datos morfológicos de los ejemplares de Craugastor yucatanensis reportados en esta nota. Simbología: Largo hocico-cloaca (LHC), ancho cabeza (AC), largo fémur (LF), largo pata (LP), diámetro del tímpano (DT), diámetro del ojo (DO).

\begin{tabular}{|c|c|c|c|c|c|c|c|c|}
\hline Número de catálogo & Localidad & Sexo & LHC & $A C$ & $\mathrm{LF}$ & LP & DT & DO \\
\hline UTADC 9547 & Izamal, Yucatán, México & N/D & - & - & - & - & - & - \\
\hline UTADC 9548 & Homún, Yucatán, México & Hembra & 36 & 14 & 17 & 16 & 3.5 & 5 \\
\hline UTADC 9549 & Homún, Yucatán, México & Macho & 30.5 & 12 & 17 & 14 & 3 & 4 \\
\hline UTADC 9550 & Homún, Yucatán, México & Macho & 31 & 12 & 18 & 14 & 3 & 4 \\
\hline UTADC 9551 & Homún, Yucatán, México & Hembra & 38 & 15 & 18 & 17 & 3 & 4.5 \\
\hline
\end{tabular}




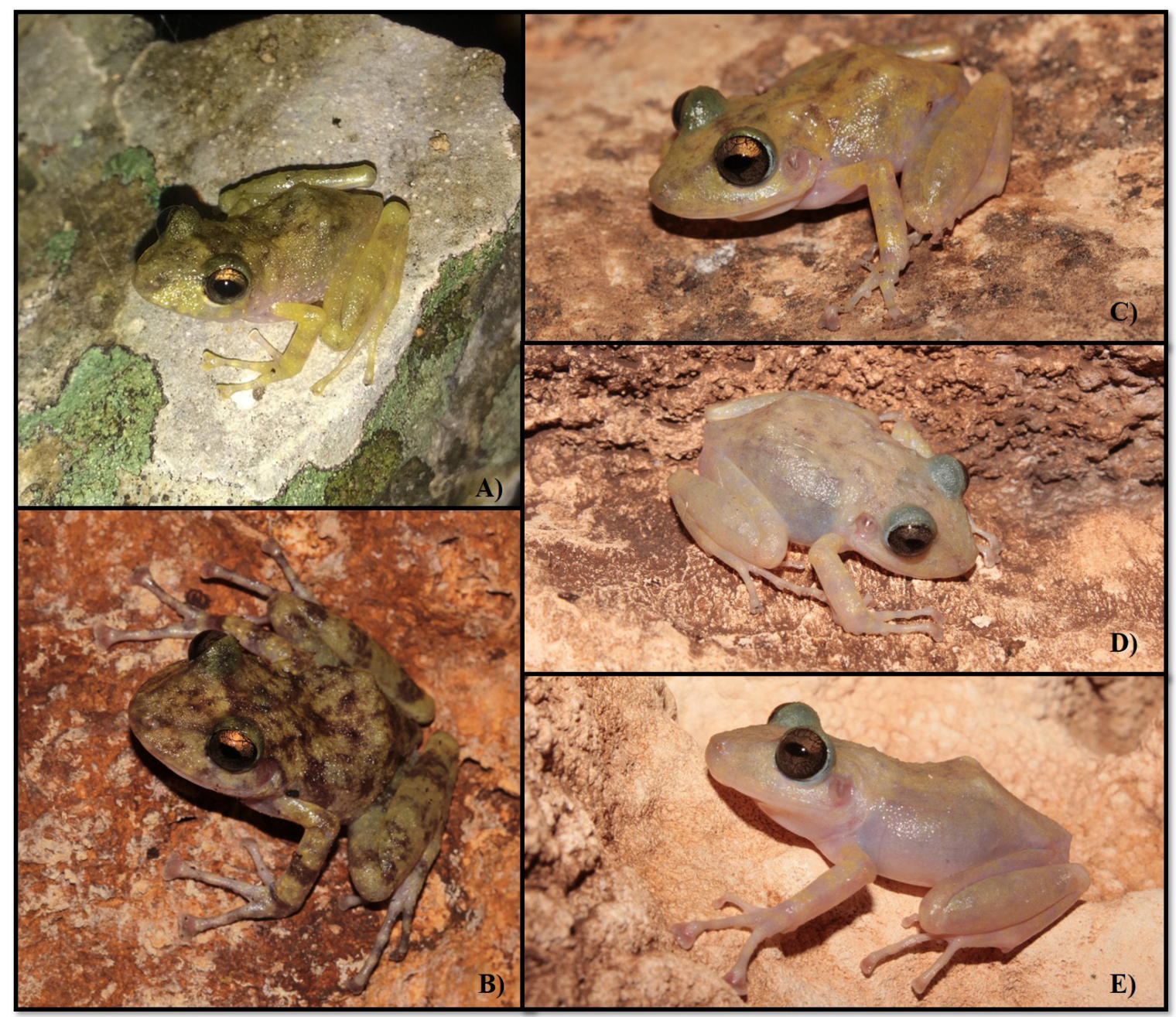

Figure 1. Specimens of Craugastor yucatanensis. Individual found in Izamal, Yucatán, (UTADC 9547) (A), Photo: Aarón Rodríguez-Blanco; Individuals found in Homún, Yucatán (UTADC 9548)(B), (UTADC 9549)(C), (UTADC 9550) (D), (UTADC 9551) (E), photo: Pedro E. Nahuat-Cervera.

Figura 1. Ejemplares de Craugastor yucatanensis. Individuo encontrado en Izamalı. Yucatán (UTADC 9547) (A), fotografía: Aarón Rodríguez-Blanco; Individuos encontrados en Homún, Yucatán (UTADC 9548) (B), (UTADC 9549) (C), (UTADC 9550) (D), (UTADC 9551) (E), fotografía: Pedro E. Nahuat-Cervera.

Table 2. Microenvironmental and microhabitat variables. Symbology: vertical position (m) (Pv), environmental temperature (Ta), environmental relative humidity (Ha), microenvironmental temperature (Tm), microenvironmental relative humidity (Hm), substrate temperature (Ts), body temperature (Tc).

Tabla 2. Variables microambientales y del microhábitat. Simbología: Posición vertical (Pv), temperatura ambiental (Ta), humedad ambiental relativa (Ha), temperatura microambiental (Tm), humedad microambiental relativa (Hm), temperatura del sustrato (Ts), temperatura corporal (Tc).

\begin{tabular}{llccccccc}
\hline Número de Catálogo & Microhábitat & Pv & Ta & Ha & Tm & Hm & Ts & TC \\
& & & & & & & & \\
\hline UTADC 9548 & Pared rocosa & $0.77 \mathrm{~m}$ & $29.9^{\circ} \mathrm{C}$ & $75.2 \%$ & $29.4^{\circ} \mathrm{C}$ & $74.1 \%$ & $23.8^{\circ} \mathrm{C}$ & $23.8^{\circ} \mathrm{C}$ \\
UTADC 9549 & Sobre roca & $0.63 \mathrm{~m}$ & $29^{\circ} \mathrm{C}$ & $73.8 \%$ & $28.8^{\circ} \mathrm{C}$ & $70.2 \%$ & $22.5^{\circ} \mathrm{C}$ & $22.1^{\circ} \mathrm{C}$ \\
UTADC 9550 & Pared rocosa & $1.42 \mathrm{~m}$ & $28.1^{\circ} \mathrm{C}$ & $74.4 \%$ & $28.1^{\circ} \mathrm{C}$ & $73.2 \%$ & $22.6^{\circ} \mathrm{C}$ & $23.3^{\circ} \mathrm{C}$ \\
UTADC 9551 & Sobre roca & $2.01 \mathrm{~m}$ & $28.9^{\circ} \mathrm{C}$ & $69.7 \%$ & $29.1^{\circ} \mathrm{C}$ & $72.7 \%$ & $23.0^{\circ} \mathrm{C}$ & $23.1^{\circ} \mathrm{C}$ \\
\hline
\end{tabular}




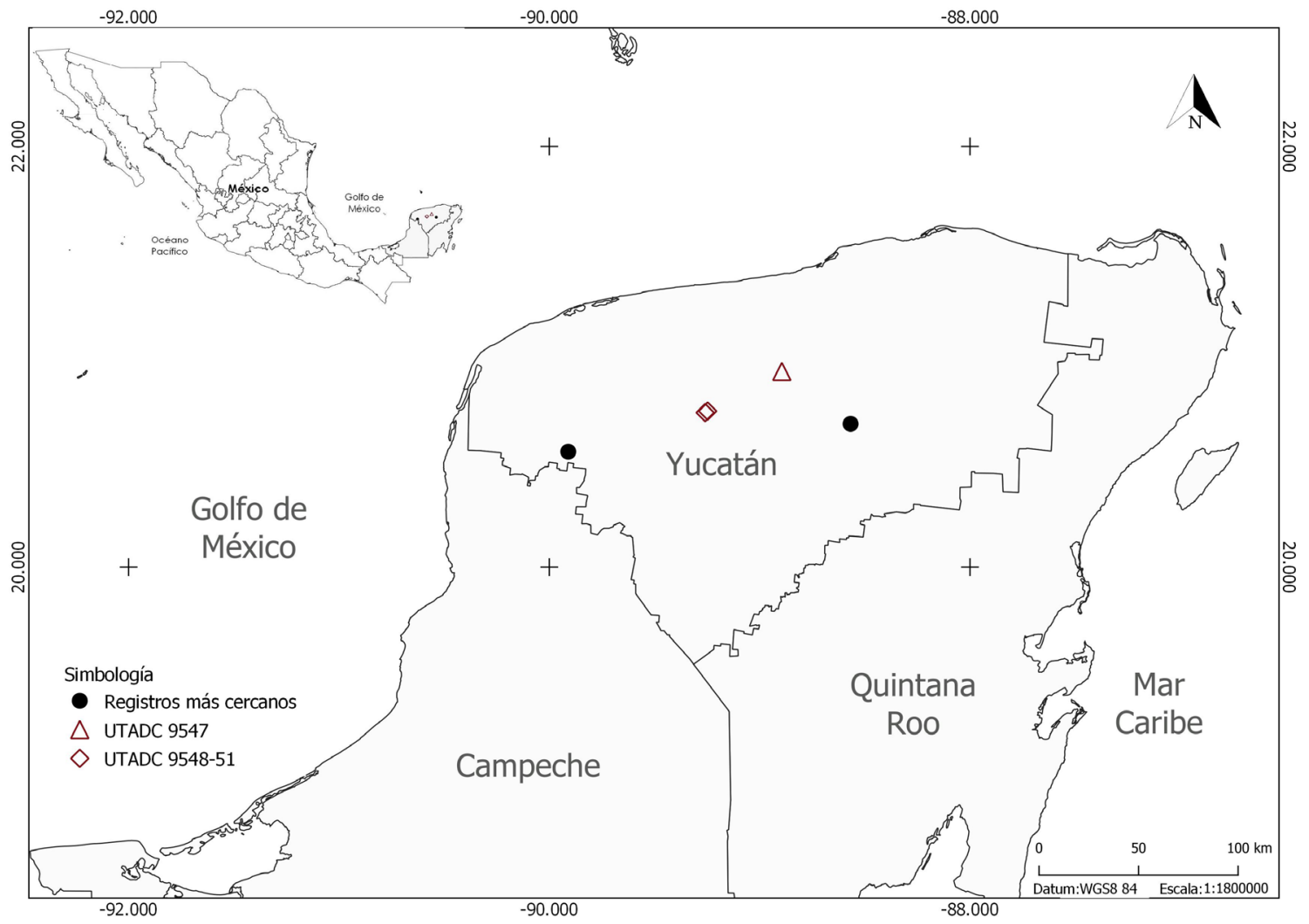

Figure 2. New records of Craugastor yucatanensis (rhombus and triangle) and nearest published records (circles).

Figura 2. Nuevos registros de Craugastor yucatanensis (rombo y triángulo) y registros publicados más cercanos (círculos).

Opichén, Yucatán (Ortiz-Medina et al., 2016) y $72.2 \mathrm{~km} \mathrm{E,} \mathrm{en} \mathrm{la}$ zona arqueológica de Chichén Itzá, Tinúm, Yucatán (CarbajalMárquez et al., 2019). De acuerdo con Carbajal-Márquez et al. (2019), se pudo determinar el sexo de los ejemplares por medio de sus medidas morfológicas, identificando dos machos y dos hembras. La vegetación que rodea a las cavernas donde se encontraron los individuos de C. yucatanensis en el municipio de Homún se compone por selva baja caducifolia y vegetación secundaria.

Agradecimientos.- Agradecemos a José Rogelio CedeñoVázquez por la verificación de los ejemplares encontrados, a Christian García-Balderas por la información brindada sobre su observación y a Gregory G. Pandelis por proporcionar los números de catálogo.

\section{LITERATURA CITADA}

Carbajal-Márquez, R.A., L.F. Díaz-Gamboa, T. Ramírez-Valverde, C.M. García-Balderas, P.E. Nahuat-Cervera \& J.R. CedeñoVázquez. 2019. Description of the male of Craugastor yucatanensis (Lynch, 1965) (Anura, Craugastoridae), its advertisement call, and additional data on females. Zookeys 900: 129-139.

Ortiz-Medina, J.A., D.E. Chan-Espinoza \& E. García-Padilla. 2016. Craugastor yucatanensis. Distribution Notes. Mesoamerican Herpetology 3:172. 